\title{
Quantitative affinity parameters of synthetic hydroxyapatite and enamel surfaces in vitro
}

Kathia Fabritius-Vilpoux Dr.

Postdoctoral Scientist, Microstructure Physics and Alloy Design,

Max-Planck-Institut für Eisenforschung GmbH, Düsseldorf, Germany

Joachim Enax Dr.

Scientific Expert Research Oral Care, Research Department, Dr. Kurt Wolff GmbH \& Co. KG, Bielefeld, Germany

Michael Herbig Dr.-Ing.

Head of Research Group/Senior Scientist, Microstructure Physics and Alloy

Design, Max-Planck-Institut für Eisenforschung GmbH, Düsseldorf, Germany
Dierk Raabe Dr.-Ing.

Professor, Head of Department, Microstructure Physics and Alloy Design, Max-Planck-Institut für Eisenforschung GmbH, Düsseldorf, Germany

Helge-Otto Fabritius Dr.

Head of Research Group/Senior Scientist, Microstructure Physics and Alloy Design, Max-Planck-Institut für Eisenforschung $\mathrm{GmbH}$, Düsseldorf,

Germany (corresponding author: h.fabritius@mpie.de)

Particulate synthetic hydroxyapatite (HAP) is rapidly gaining importance as a biomimetic agent in oral care products. The prerequisite for an adequate effect of the treatment is an efficient attachment of the HAP particles to the tooth substance. However, quantitative data about the interaction and affinity parameters involved are scarce. The authors used an in vitro approach with standardized bovine tooth enamel surfaces and aqueous dispersions of chemically pure synthetic HAP particles as a model system. Structural characterization of the HAP powder using high-resolution scanning electron microscopy (SEM) shows that the particles are micrometer-sized stable clusters of crystallites that closely resemble the structure of natural enamel. Using SEM image analysis, the authors investigated the influence of HAP particle concentration and particle size on the efficiency of attachment to bovine tooth enamel based on pure mineral-mineral interaction. The results show that both play an important role and can be tailored for optimizing the efficiency of corresponding oral care formulations. The results also reveal the presence of structures resembling mineral bridges at the interfaces between HAP particles and enamel that provide indications for possible interaction mechanisms.

\section{Notation}

$A_{\mathrm{r}} \quad$ aspect ratio

$d_{\max } \quad$ crystallite length

$d_{\min } \quad$ crystallite width

$N_{\text {particles }}$ number of particles

\section{Introduction}

Human teeth consist of bone-like dentin covered with an external layer of enamel that is essential for nutrition in constituting a hard tissue with properties tailored for efficiently biting and grinding food items. Healthy enamel consists of $c$. 97\% hydroxyapatite (HAP), $\mathrm{Ca}_{5}\left(\mathrm{PO}_{4}\right)_{3}(\mathrm{OH})$, in the form of thin elongated crystallites in the nanometer size range. These crystallites form tightly packed bundles with a highly organized spatial arrangement. This so-called prismatic enamel is extremely hard and at the same time highly fracture resistant. ${ }^{1,2}$ The enamel layer is formed in a genetically controlled biomineralization process before the tooth erupts. $^{3,4}$ Unlike bone, the enamel is a static tissue after its maturation and cannot be regenerated naturally. Therefore, modern oral care focuses on the protection and preservation of enamel with a large spectrum of treatment strategies relying mostly on fluorides for everyday care ${ }^{5,6}$ and ceramics for repair and replacement purposes. ${ }^{7,8}$ Despite these efforts, almost the whole world population is affected by negative enamel conditions with the major factors being caries and excessive erosion, which are mainly promoted by modern dietary habits. ${ }^{9-11}$ In addition to the established methods, bioinspired concepts that rely on mimicking the natural structures, ${ }^{12,13}$ protective agents and preventive effects ${ }^{14-17}$ have therefore received increasing attention in modern oral care and dentistry within the past years. In the field of oral care, the use of particulate HAP as an active ingredient is on the advance because of its chemical and structural similarity to dental enamel. ${ }^{18-22}$

Oral care formulations based on particulate HAP have been shown to be efficient in addressing a number of clinical indications such as prophylaxis against caries ${ }^{23,24}$ and protection from dentin hypersensitivity by the occlusion of open dentin tubuli. $^{25-28}$ Additional effects are, for example, the improvement of periodontal health, ${ }^{29}$ the formation of a protective layer on enamel, ${ }^{30,31}$ biofilm management ${ }^{16,32}$ and tooth whitening. ${ }^{33}$

The necessary condition for HAP particles to take effect is that they come into contact with the tooth surface. It has been shown that pure HAP particles attach to pellicle-covered enamel surfaces under in situ conditions without using any additional additives such as polymers. With increasing time, however, a partial desorption of HAP particles was observed. ${ }^{16}$ In vitro studies also confirmed HAP particles adhering to enamel surfaces, where they might contribute to tooth whitening. ${ }^{33,34}$ The by far most interesting and debated question is whether and how particulate HAP contributes to the natural enamel remineralization process and, depending on this, its potential to aid in repairing, for example, lesions caused by caries or erosion as well as its ability to form a biomimetic coating. ${ }^{35-37}$ In this context, the group of Yamagishi et $a l^{38}$ reported on a HAP paste for rapid tooth repair where an enamel-repairing effect was demonstrated by transmission electron microscopy (TEM) and atomic force microscopy. The remineralization of early enamel caries lesions was 
studied in vitro using different concentrations of particulate HAP by Huang et al. ${ }^{39}$ It was found that for this purpose, 10\% HAP might be most efficient. Remineralizing effects similar to those induced by fluorides were observed in situ using microradiography. ${ }^{40}$ An ex-in vivo study by Lelli et $a l^{30}$ demonstrated the formation of a biomimetic coating on enamel surfaces after application of a HAPcontaining toothpaste. However, other studies failed to detect an inhibition of caries-mediated demineralization in vitro. ${ }^{41}$

To date, the interaction mechanisms between HAP particles and enamel surfaces that lead to the remineralization phenomenon are not yet understood in detail. ${ }^{33}$ A possible mechanism currently under discussion is that HAP particles may induce crystallization by functioning as nuclei that attract calcium and phosphate ions from saliva. ${ }^{19}$

While the direct interaction of HAP particles with enamel and pellicle surfaces is qualitatively well proven, ${ }^{16,21,30,31,33,34}$ only very little is known about the influence of quantitative parameters on HAP applications. The main parameters expected to influence potentially the efficacy of HAP as an active ingredient in oral care products are concentration, particle size and additives that may influence the adhesion properties. For fluoride-based toothpastes, it has been shown that the caries prophylactic effect increases with fluoride concentration. ${ }^{42}$ For particulate HAP, understanding the dose-response relationship of attachment is crucial for optimizing the efficiency of corresponding oral care approaches. The particle size is an important factor for adhesion efficiency, which is supported by experimental data indicating that adhesion of smaller particles to enamel is favored. ${ }^{34}$ Finally, the qualitative studies available indicate that HAP particles adhere better if organic material is present such as in enamel covered with the proteinaceous pellicle and dentin, which has a much higher organic material content (about 20\%) than enamel (about 1.5\%). ${ }^{43,44}$

The aim of this study is to bridge this gap and provide quantitative information about the correlation of concentration and particle size with adhesion efficiency between chemically pure synthetic particulate HAP and polished organic-free enamel surfaces in vitro. For this, the authors prepared standardized bovine enamel surfaces that allow studying the pure mineral-mineral interaction without the influence of individual patient-related parameters or additives. The results of the adhesion experiments were analyzed using highresolution scanning electron microscopy (SEM), followed by a comprehensive quantitative analysis based on images obtained from randomized sample locations. The results are expected to shed light on the interaction mechanisms between particulate HAP and tooth enamel and provide parameters that can be used to optimize the efficiency of HAP-based oral care formulations.

\section{Materials and methods}

\subsection{HAP powder characterization}

For this study, the authors used a chemically pure HAP powder that is commercially available. To assess the similarity of the material to human dental enamel and to characterize the particle size distribution in the powder, a structural analysis was performed using high-resolution SEM. For this, a thin layer of HAP powder was spread on aluminum SEM sample holders covered with self-adhesive carbon foil pads. The samples were sputter-coated with a $4 \mathrm{~nm}$ thick layer of platinum (Gatan Precision Etching Coating System 682) prior to inspection with a Zeiss Crossbeam 1540 XB scanning electron microscope. SEM images of randomly chosen sample areas were used to determine semiquantitatively the dimensions of the particles' crystallite subunits (length, width, aspect ratio). A set of three sample images recorded at $\times 2000$ magnification was used for quantitative particle size determination following the procedure used for the adhesion experiments (see Section 2.4 and Figure S10 in the online supplementary material). A clinically extracted permanent human premolar was used as a reference sample for crystallite structure. The tooth surface was gently etched with phosphoric acid $(0 \cdot 07 \%, 20 \mathrm{~s})$ to remove the pellicle layer and expose the HAP crystallites. After being washed in demineralized water $(5 \mathrm{~s})$, the sample was mounted to an aluminum SEM sample holder and air-dried overnight. Subsequently, the tooth was sputter-coated with $4 \mathrm{~nm}$ platinum before inspection through SEM.

\subsection{Bovine tooth enamel model}

Complete lower jaws of domestic cattle were acquired from a local meat producer with their own breeding and processing facilities (Laame $\mathrm{GmbH} \&$ Co. KG, Wuppertal, Germany). The jaws originate from male animals aged 24 months and were obtained fresh. In the next step, all teeth were extracted intact and thoroughly cleaned from any adhering tissue and bone. The root tips were cut off to clean also the root channels and pulp chambers. Subsequently, the teeth were stored in $0 \cdot 1 \%$ aqueous thymol solution ${ }^{45}$ in order to avoid any unwanted effects from desiccation or bacterial decay of the organic phase. For the experiments, the authors used the incisors and canines due to their geometrical and morphological resemblance to human teeth. To obtain flat samples with a maximum of enamel surface area, each tooth was clamped using the root and consecutively cut four times using a water-cooled cutting disk (Buehler, IsoMet 5000, linear precision saw, 3000 revolutions per minute $(\mathrm{rpm}), 2 \mathrm{~mm} / \mathrm{min}$; Figure 1(a)): perpendicular and along the median line, followed by two transversal cuts. This yielded a total of eight tooth pieces with roughly similar dimensions, four exposing the labial surface and four exposing the lingual surface (Figure 1(b)). All samples were stored in $0 \cdot 1 \%$ aqueous thymol solution until further processing. The preparation steps necessary to obtain enamel surfaces of high and reproducible quality were all carried out consecutively, including the application of HAP dispersion, to exclude unwanted effects from desiccation or interim storage. In the first step, the samples were washed in demineralized water to remove adhering thymol solution. Then, the inner surface of every tooth piece was ground manually using dry 600 grit abrasive paper until the outer enamel surface and inner dentin surface formed parallel planes (Figure 1(c)). These flat sample pieces were fixed on standard aluminum SEM holders using instant 


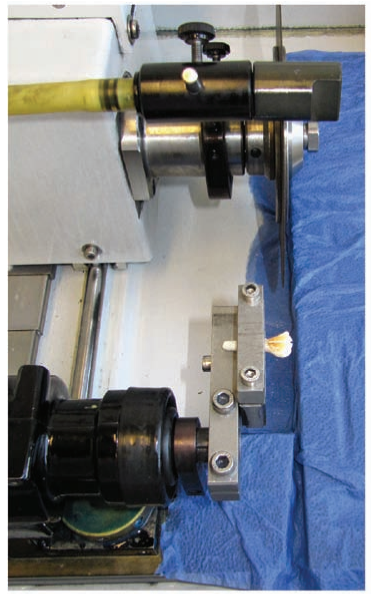

(a)

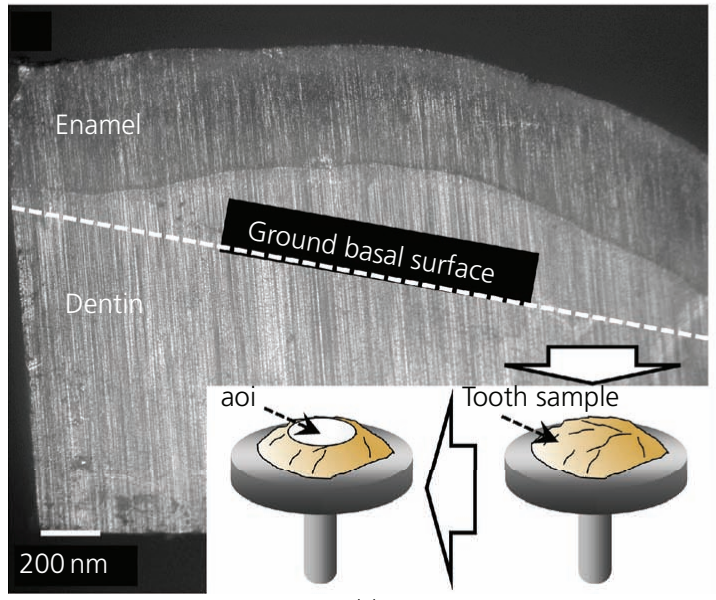

(c)

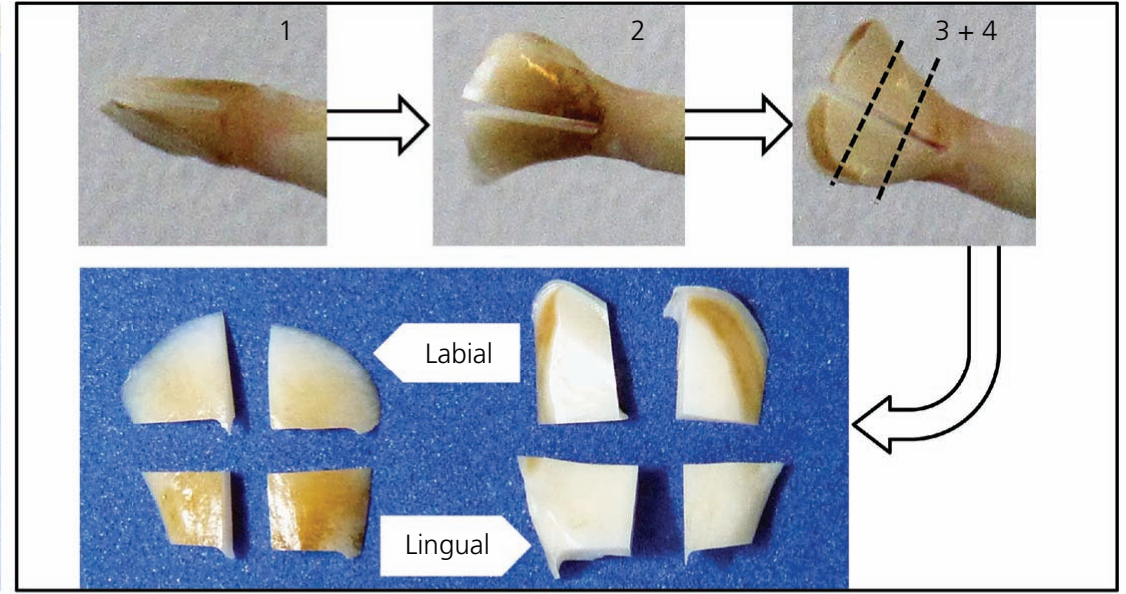

(b)

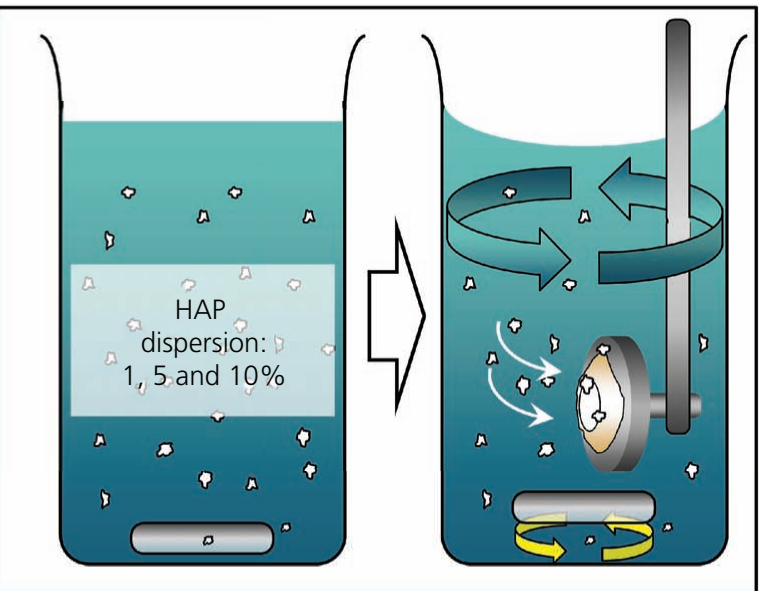

(d)

Figure 1. (a) A bovine incisor mounted in the cutting device. (b) Sequential cuts (1-4) were performed to obtain eight flat enamel samples. (c) Cross-section of a tooth sample showing the enamel layer on top of the inner dentin. The lower sample edge to be ground is indicated by the dashed line. The insert schematically indicates the next preparation steps where the samples are mounted and the area of interest (aoi) is polished. (d) Schematic depiction of the mouth rinse-inspired procedure used to apply the HAP particles to the enamel surface

adhesive. Subsequently, the exposed enamel surface of each sample was carefully polished on a water-cooled rotating lab grinder parallel to the sample holder's surface at $150 \mathrm{rpm}$ using 2500-4000 grit abrasive papers (Figure 1(c)). After a quick cleaning step with water, all samples were polished using a textile-covered disk with $1 \mu \mathrm{m}$ diamond particle suspension. The final polishing step was carried out using several drops of a silica particle ( $\sim 50 \mathrm{~nm}$ dia.) suspension alone for $\sim 30 \mathrm{~s}$, after which the process was continued under continuous water flow. Subsequently, the samples were thoroughly cleaned with $100 \%$ ethanol and subjected to a $30 \mathrm{~s}$ cleaning step in an ultrasound bath submersed in ethanol. The final preparation step was to etch the polished surface gently for $5 \mathrm{~s}$ in $0.07 \%$ phosphoric acid, after which the samples were submerged in demineralized water for $5 \mathrm{~s}$. Excess water was removed using tissue paper. After this, each sample was subjected to the adhesion experiment immediately to avoid desiccation and thus the formation of cracks in the polished surface.

\subsection{Adhesion experiments}

Three dispersions containing 1, 5 and $10 \mathrm{wt} \%$ of HAP powder were prepared using demineralized water $(\mathrm{pH} 7 \cdot 0)$ without any additives and aliquoted in $250 \mathrm{ml}$ glass beakers. The highest concentration was limited to $10 \%$ because this quantity corresponds to the amount of HAP commonly found in various commercial oral care mouth rinse formulations. For the adhesion experiments, the authors used three polished samples originating from different animals and teeth for each concentration. Each sample was exposed to the dispersion for $1 \mathrm{~min}$ under continuous stirring at $\sim 100 \mathrm{rpm}$ such that the polished surface was oriented perpendicular to the flow direction. Thus, the particles in the dispersion were enabled to impact the sample surface directly, simulating the application procedure of a commercial mouth rinse (Figure 1(d)). Subsequently, the samples were submerged in demineralized water for $5 \mathrm{~s}$ to remove loose material followed by a quick wash in $100 \%$ methanol to remove the water. Then, the samples were dried in a warm, gentle air stream and prepared for SEM analysis. 
Bioinspired, Biomimetic and Nanobiomaterials Volume 8 Issue BBN2
Quantitative affinity parameters of synthetic hydroxyapatite and enamel surfaces in vitro

Fabritius-Vilpoux, Enax, Herbig, Raabe and Fabritius

\subsection{SEM documentation and image analysis}

The air-dried enamel samples were sputter-coated with $4 \mathrm{~nm}$ of platinum using a Gatan PECS 682 coating device. Subsequently, all samples were inspected using a Zeiss Crossbeam $1540 \mathrm{XB}$ focused ion beam SEM microscope. To randomize the choice of areas for quantitative analysis, each sample was first inspected at $\times 45$ magnification. For the samples treated with 1 and 5\% HAP, five approximately evenly distributed areas were chosen that were digitally marked on the recorded image, and for the samples treated with $10 \%$ HAP, three (panels A and B of Figures S1-S9 in the online supplementary material). Subsequently, the coordinates of the chosen enamel areas of interest were saved and images at a magnification of $\times 10000$ were recorded at each location. Only in cases where an obvious defect such as a large crack in the enamel was situated in the image, the imaging site was shifted to adjacent defect-free areas. All images were saved at a pixel resolution of $1024 \times 768$ and further used to mark the particles for quantitative analysis. For this, the authors used the software Adobe Photoshop CS5 Extended, version 12.1 (Adobe Inc.). Using selection tools and image layers, the particles were manually marked to generate a color mask of their planar projections. The number of pixels of each image was constant, and the absolute surface area of an individual pixel could be calculated using the scale bar length ported from the SEM software. Thus, the area occupied by the planar projection of each HAP particle could be quantified. The image analysis tools integrated in Photoshop were used to automatically determine the number of individual particles and the area that each particle occupied for each sampled location to obtain the particle size distribution of both the used HAP powder stock and the adhering particles after application of the dispersions, respectively. Since the use of area $\left(\mu \mathrm{m}^{2}\right)$ as a unit of particle size is not intuitive, these values were geometrically converted to particle diameter $(\mu \mathrm{m})$ where necessary. Under the simplified assumption that the particles are spherical, their circular area represented by the planar projection was used to calculate the corresponding diameter. From the pooled particle data, the degree of coverage was calculated for every HAP concentration assessed by simple subtraction of the area occupied by particles from the total image area that represents the enamel surface background. For preparation of the figures, brightness and contrast of the SEM micrographs were adjusted using Adobe Photoshop CS5 Extended, version 12.1 (Adobe Inc.) where necessary. The graphs and statistical analysis were prepared using Excel 2010 (Microsoft) and OriginPro 8.6 (OriginLab Corporation).

\section{Results}

\subsection{Structural characterization of synthetic HAP particles}

The individual particles constituting the used synthetic HAP powder consist of small, rod-shaped HAP crystallites that cluster to form micrometer-sized particles (Figure 2(a)). The crystallites have relatively uniform dimensions with a thickness of about $30 \mathrm{~nm}$ and a length of about $80 \mathrm{~nm}$, resulting in an aspect ratio of 0.38 (Table 1). Within a particle, the crystallites are densely packed without an apparent preferential spatial orientation (Figure 2(a)). The HAP crystallites observed in cleaned human (Figure 2(b)) and bovine enamel (Figures 5 and 7) have similar

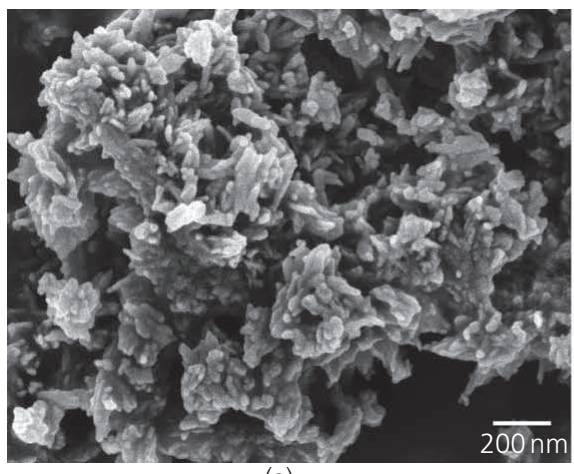

(a)

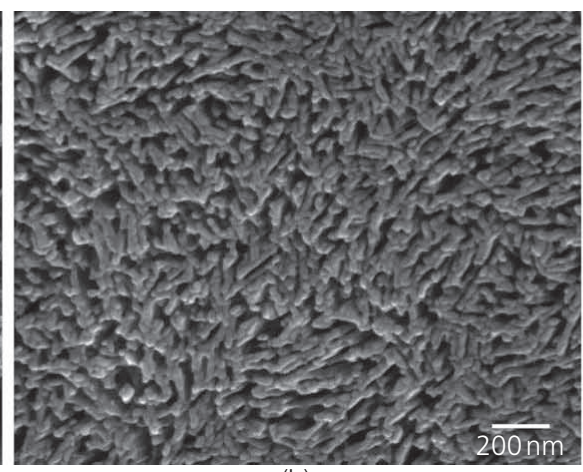

(b)

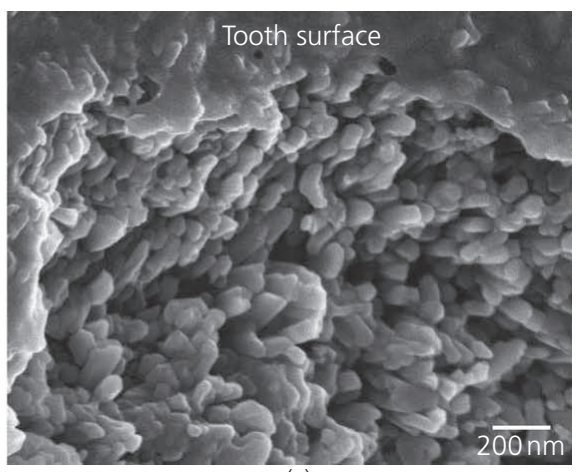

(c)

Figure 2. SEM micrographs showing the microstructure of (a) the HAP microclusters used for the adhesion experiments in comparison with the microstructures of (b) human tooth enamel surfaces gently etched with phosphoric acid and (c) fractured human enamel just below the tooth surface. (b) and (c) are both samples from human permanent premolars

Table 1. Size and shape of crystallites constituting the HAP powder used for the adhesion experiments (calculated from 51 crystallites; based on SEM images)

\begin{tabular}{lccc} 
& Crystallite length $\boldsymbol{d}_{\text {max }}: \mathbf{n m}$ & Crystallite width $\boldsymbol{d}_{\text {min }}: \mathbf{n m}$ & Aspect ratio $\left(\boldsymbol{A}_{\mathbf{r}}=\boldsymbol{d}_{\text {min }} / \boldsymbol{d}_{\text {max }}\right)$ \\
\hline Mean with SD & $81.6 \pm 30.7$ & $30.6 \pm 9.3$ & 0.38 \\
Median & 80 & 30 & 0.38
\end{tabular}

SD, standard deviation 
Bioinspired, Biomimetic and Nanobiomaterials Volume 8 Issue BBN2
Quantitative affinity parameters of synthetic hydroxyapatite and enamel surfaces in vitro

Fabritius-Vilpoux, Enax, Herbig, Raabe and Fabritius thicknesses. Their lengths are difficult to determine since their anisotropic parallel arrangement in both prismatic and interprismatic enamel does rarely permit observing where an individual crystallite ends. In fractured untreated human enamel close to the tooth surface, the crystallites are thicker, but their lengths roughly correspond to the synthetic ones (Figure 2(c)).

The crystallite particles have irregular shapes with a tendency of being roughly spherical (Figures 3(a) and 3(b)), and their size greatly varies within the powder (Figure 3(a)). Individual nanoscopic crystallites were very only exceptionally observed in the powder samples (Figure 3(b)). To account for the broad particle size distribution of the synthetic HAP powder, the images used for quantitative particle size determination were recorded at a lower magnification $(\times 2000$, Figures 3 (a) and S10 in the online supplementary material) than those used for the adhesion experiment evaluation $(\times 10000$, Figures 4 and $\mathrm{S} 1-\mathrm{S} 9$ in the online supplementary material). Thus, particles in the smaller size range are not well resolved. However, their presence is confirmed by high-resolution SEM analysis of the native powder (Figure 3(b)) and the results of the adhesion experiments (Figures 4 and S1-S9 in the online supplementary material). Figure 3(c) shows the distribution of different particle sizes as a percentage of the total particle number ( $N_{\text {particles total }}$ 16499)

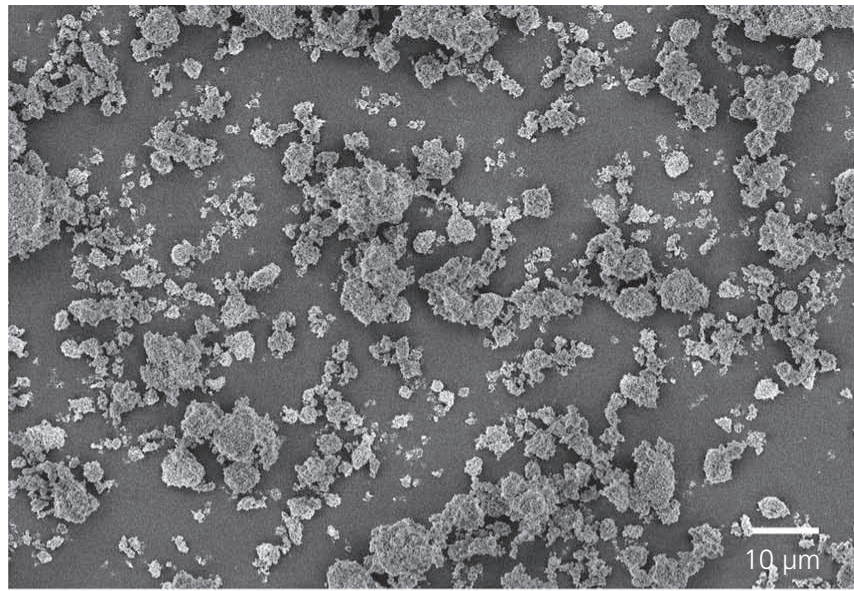

25

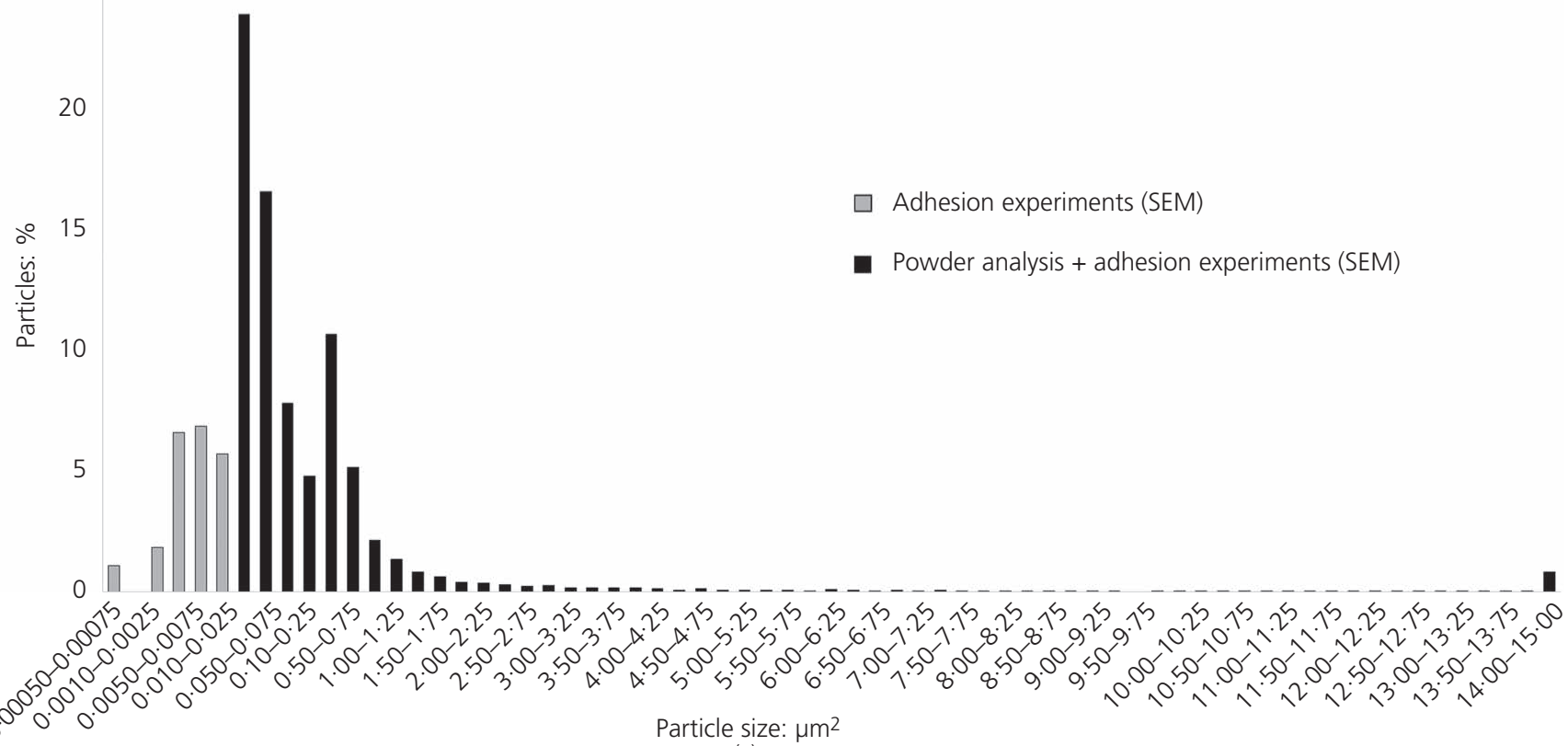

(c)

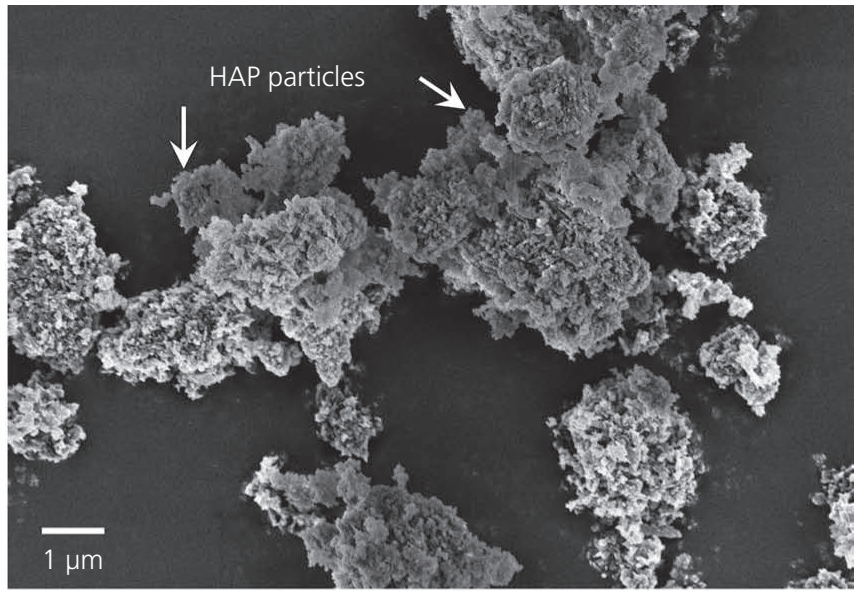

(b)

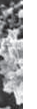


Quantitative affinity parameters of synthetic hydroxyapatite and enamel

surfaces in vitro

Fabritius-Vilpoux, Enax, Herbig, Raabe and Fabritius

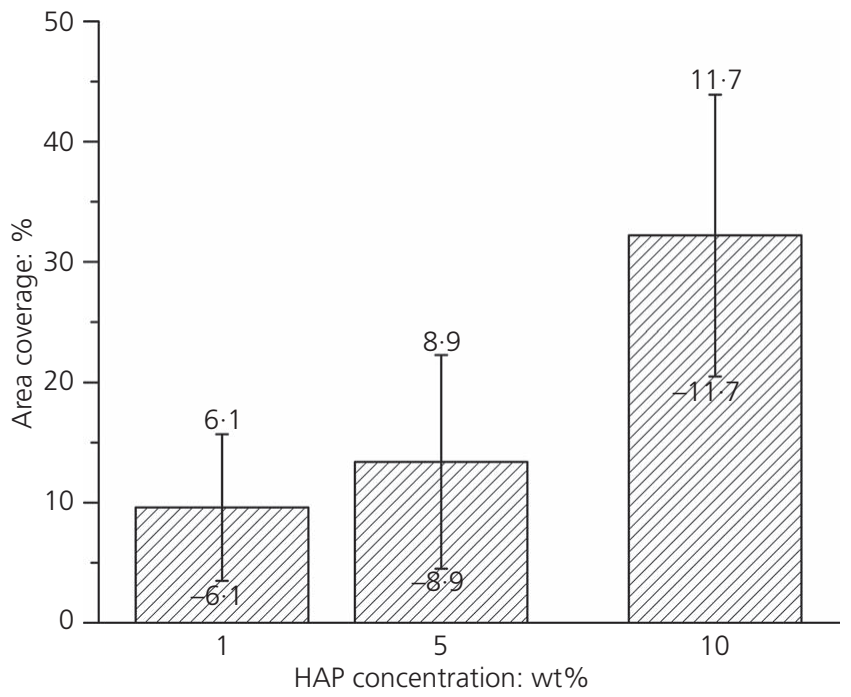

Figure 4. Dose-response relationship of HAP particle adhesion to standardized bovine tooth enamel surfaces in vitro. Fifteen areas distributed on three different enamel samples were analyzed for the 1\% ( $\left.N_{\text {particles: }}: 2345\right)$ and $5 \%$ HAP dispersions ( $\left.N_{\text {particles: }}: 5912\right)$, and nine areas distributed on three different samples for the $10 \%$ HAP dispersion ( $N$ particles: 6006$)$. Each analyzed area had a size of $453 \cdot 12 \mu \mathrm{m}^{2}$. Coverage is expressed as percentage of the total analyzed area with standard deviations

allotted to different size classes. The graph contains both particles analyzed from powder samples and those analyzed from the adhesion experiments. The area projections of the smallest particles observed cover $500-750 \mathrm{~nm}^{2}$, corresponding to diameters of roughly $25-30 \mathrm{~nm}$. It must be noted that this size range represents the resolution limit of this study's quantitative analysis, since in this size range a particle is rarely represented by more than a single pixel. The largest particles encountered had an area projection of just below $1500 \mu \mathrm{m}^{2}$, corresponding to a diameter of about $40 \mu \mathrm{m}$. The majority of the particles show a Gaussian distribution and have area projections between $2500 \mathrm{~nm}^{2}$ and $1.25 \mu \mathrm{m}^{2}$, corresponding to diameters ranging from about $60 \mathrm{~nm}$ to about $1.3 \mu \mathrm{m}$. The most abundant class of particles in the powder have area projections between 0.01 and $0.025 \mu^{2}$, corresponding to diameters between about 110 and $180 \mathrm{~nm}$. This particle class represents $\sim 24 \%$ of all analyzed particles and forms the maximum of the distribution curve. The other abundant size classes all make up at least $2 \%$ of the total particle count. Particles below $2500 \mathrm{~nm}^{2}$ and above $1.5 \mu \mathrm{m}^{2}$ are rare, and each of their size categories represents less than $1 \%$ of the total particle count (Figure 3(c)). Solely the very large particles are notably abundant with a fraction of about $1 \%$.

\subsection{Quantitative analysis of the affinity of HAP particles to enamel surfaces}

\subsubsection{Concentration dependence of particle adhesion}

The evaluation of the adhesion experiments shows that the degree of coverage of the enamel surfaces increases with increasing concentration of the applied HAP particle dispersion. Application of the HAP dispersions resulted in an average total enamel area coverage of $9 \cdot 6 \pm 6 \cdot 1 \%$ for $1 \%$ HAP concentration, $13 \cdot 4 \pm 8 \cdot 9 \%$ for $5 \%$ HAP concentration and $32 \cdot 2 \pm 11.7 \%$ for $10 \%$ HAP concentration (Figure 4). Figure 5 shows representative images of enamel samples treated with the different dispersions. The SEM analysis shows that, independent of the used concentrations, the coverage of the sample surfaces was not uniform (Figures $\mathrm{S} 1-\mathrm{S} 9$ in the online supplementary material). This is reflected by the high standard deviations obtained. With HAP concentrations increasing from 1 to $10 \%$, the standard deviation of the covered area percentage, however, decreases (Figure 4), and the particle coverage density of the sampled areas becomes more uniform (Figures S1-S9 in the online supplementary material).

\subsubsection{Size distribution of adhering particles}

The results of the adhesion experiments were used to determine the size distributions of the particles that remained on the enamel sample surfaces after application of the different HAP dispersions and the following preparation steps. Compared to the particle size distribution of the used powder (Figure 3(c)), the most obvious difference is that for all three concentrations, very large particles are virtually absent. The adhering particles from the largest class $\left(14-1500 \mu^{2}\right.$ ) represent only $0 \cdot 17 \%$ for the $1 \%$ HAP dispersion, $0 \cdot 1 \%$ for the $5 \%$ dispersion and $0 \cdot 16 \%$ of the total particle number for the $10 \%$ dispersion. In the used powder, $0 \cdot 81 \%$ of all particles belong to the largest class. As described for the powder, the lower size boundary for adhering particles is about $30 \mathrm{~nm}$ as calculated from the area projections of the smallest particle class $\left(500-750 \mathrm{~nm}^{2}\right)$. The largest particles still adhering in significant numbers belong to the class with area projections between 1 and $1.25 \mu \mathrm{m}^{2}$, which corresponds to a maximal diameter of roughly $1 \cdot 3 \mu \mathrm{m}$. Between the two threshold values, the sizes of the adhering particles show a Gaussian distribution with the largest number of particles belonging to the class with area projections between 0.01 and $0.025 \mu \mathrm{m}^{2}$ (diameters about 110 and $180 \mathrm{~nm}$ ) for all three used HAP concentrations. The second highest abundance was observed for particles with area projections between $0 \cdot 1$ and $0.25 \mu^{2}$ (diameters about 400 and $650 \mathrm{~nm}$ ), which represent 13 and $11 \%$ of all adhering particles in the samples treated with 1 and 10\% HAP dispersions, respectively. For the 5\% HAP dispersion, these particles represent only a fraction of $8 \%$. Overall, the results obtained for the three different HAP concentrations differ for each particle size class in the range of a few percent. The general trend, however, is similar and reflects the size distribution observed in the dry powder (Figure 3(c)) for particles with area projections above $0.01 \mu \mathrm{m}^{2}$. In the samples treated with 1 and 5\% HAP dispersions, virtually all observed particles adhere to the enamel surface directly (Figures 4(a) and 4(b) and S1-S6 in the online supplementary material). Solely in the samples treated with the 10\% HAP dispersion did the authors observe a tendency of the particles to form larger clusters, meaning a small fraction of the particles adhere to other particles already situated on the enamel surface. 
Bioinspired, Biomimetic and Nanobiomaterials Volume 8 Issue BBN2
Quantitative affinity parameters of synthetic hydroxyapatite and enamel surfaces in vitro

Fabritius-Vilpoux, Enax, Herbig, Raabe and Fabritius
Raw SEM images

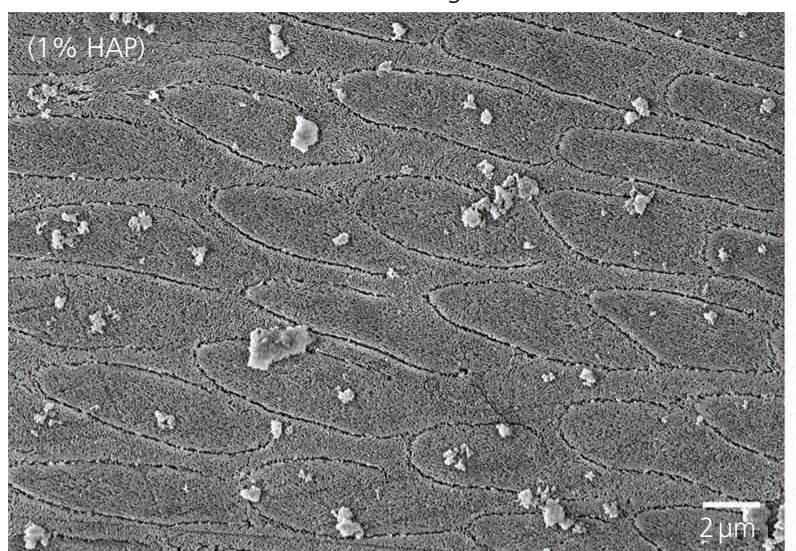

(a)

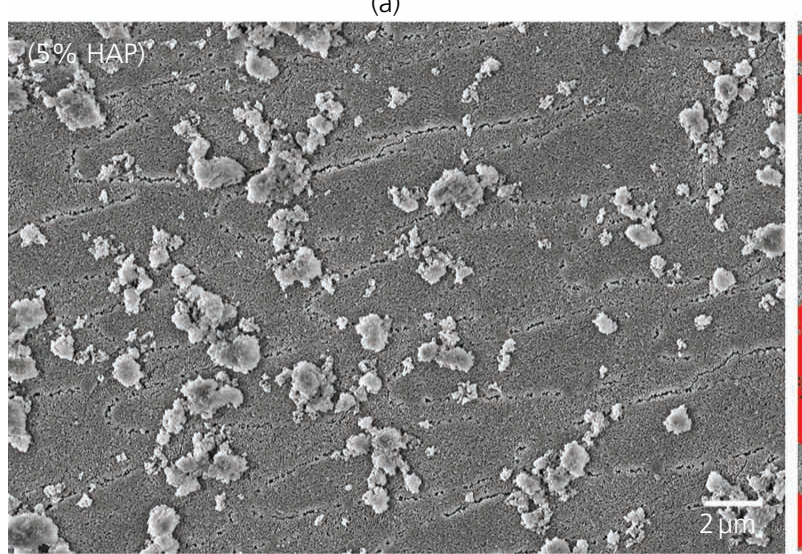

(b)

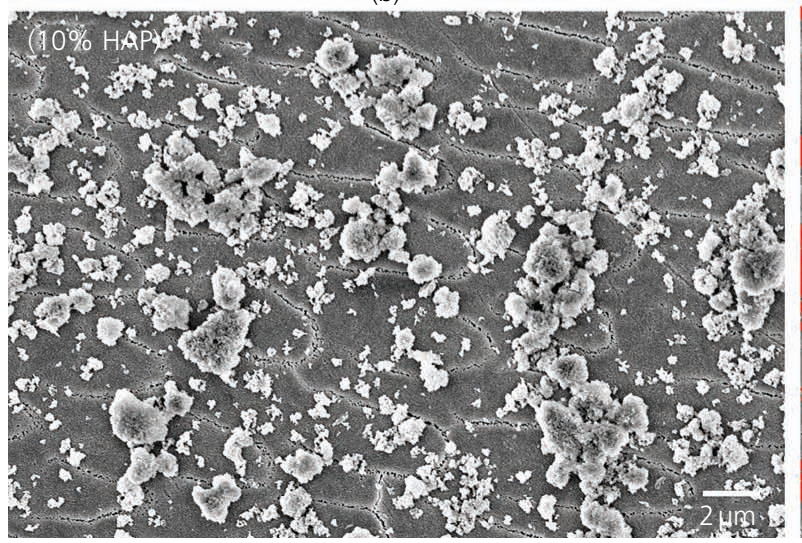

(c)
SEM images processed for particle analysis

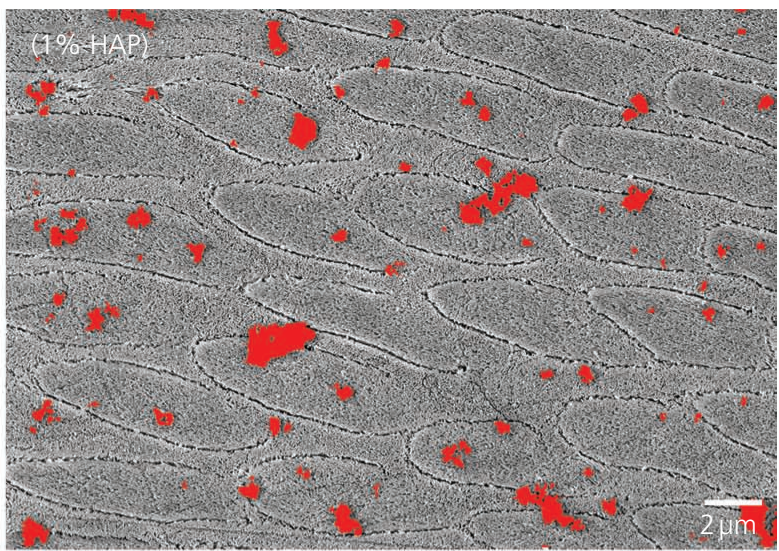

(d)

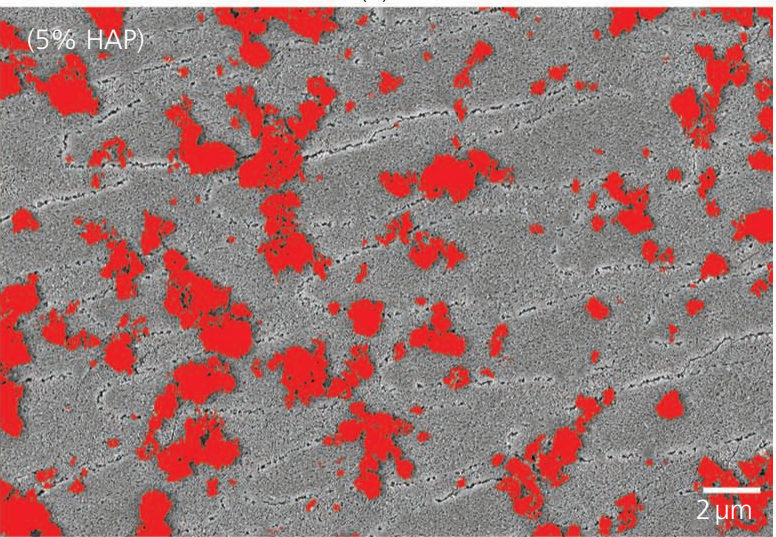

(e)

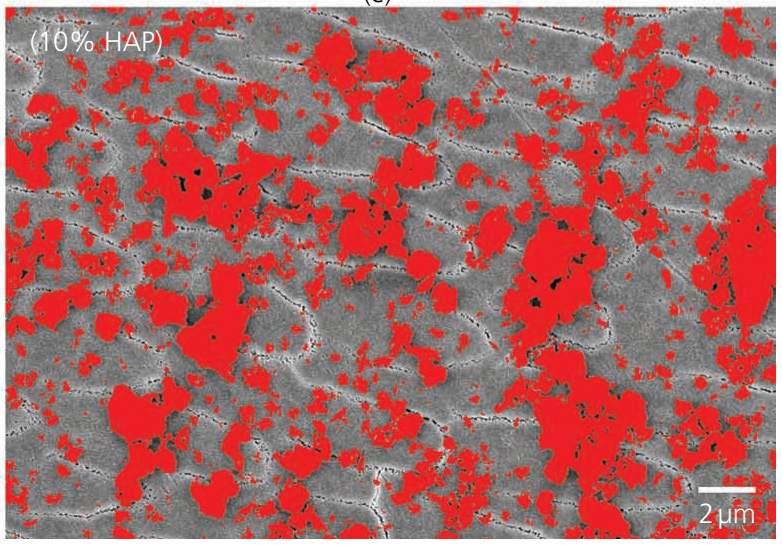

(f)

Figure 5. Representative SEM micrographs of the standardized bovine enamel sample surfaces after treatment with dispersions containing (a) 1, (b) 5 and (c) 10\% HAP particles from the same powder stock. (d), (e) and (f) show images of the same areas, respectively, with the particles marked that were used for determination of coverage and particle size distribution. Images of all analyzed samples are shown in Figures S1-S9 in the online supplementary material

\subsection{Structural characterization of the interface} between enamel and adhering HAP particles

High-resolution SEM was used to analyze the interface between the standardized bovine enamel surfaces and the adhering HAP particles (Figure 7). The results obtained on the flat samples inspected from above show that at the scale of observation, there is no recognizable interphase present between particles and surface (Figure 7(a)). This is confirmed at very high magnifications, where the crystallite clusters were observed touching the crystallites of the enamel directly (Figure 7(b)). Since in this configuration the actual interface is concealed, the authors also inspected the margins of the enamel occurring at the dentin-enamel junction occasionally 
present in the samples. Here, the dentin surface was recessed with respect to the enamel surface. This is an artifact from the polishing process caused by the lower hardness of dentin, which leads to a quicker abrasion compared to the harder enamel. Particles adhering to these sloped enamel areas were inspected at an oblique angle, allowing observing their interface with the enamel (Figure 7(c)). While the absence of foreign substances was confirmed, the authors frequently observed connections between particle structures and surface structure that resemble HAP crystallites in shape and dimensions. It was not possible to discern whether these are crystallites originally belonging to either HAP particles or the enamel surface that fused at their contact areas or connecting mineral 'bridges' that grew between neighboring crystallites during or after the application of the HAP dispersion.

\section{Discussion}

To understand the role that particulate HAP can play in the natural remineralization process and thus how it functions as a biomimetic active ingredient in oral care products, detailed knowledge is needed on how it interacts with tooth enamel, ideally with that of human teeth. A systematic quantitative in vitro study requires a large number of samples with constant quality. Since healthy human teeth are hard to acquire, the authors decided to use bovine teeth as basic material for enamel samples. The structure and properties of bovine teeth, particularly of the incisors, are very similar to human teeth. ${ }^{46}$ They are an established model material used for both in vitro ${ }^{31}$ and in situ ${ }^{16,32}$ studies. With the authors' preparation protocol, relatively large flat areas of exposed enamel are achieved that are virtually free of contaminations in the form of organic residues (e.g. pellicle residues) and of diamond and silica particles from grinding and polishing. A number of studies have shown that HAP particles adhere well to enamel surfaces. ${ }^{16,21,30,31,33,34,38,40}$ However, the in vitro studies used enamel surfaces that were covered with an artificial organic pellicle layer or applied HAP in a form where the mixture contained additional organic ingredients. In the in situ studies, the cleaned test specimens inevitably develop a pellicle layer due to their exposure to saliva. Thus, it is difficult to assess whether the HAP particles adhere to the enamel due to intrinsic mineral properties or because proteins and other organic molecules act as bonding agents. Therefore, the authors chose to use clean enamel specimens and aqueous HAP dispersions with neutral $\mathrm{pH}$ and studied particle adhesion based on pure mineral-mineral interaction.

An important prerequisite for a biomimetic oral care agent is its similarity to the target substance, in this case tooth enamel. Highresolution SEM inspection of the commercially available HAP powder that the authors used shows that the particles are actually clusters consisting of crystallites that resemble those constituting the smallest building blocks of both human (Figure 2(b)) and bovine (Figure 7(a)) enamel. The crystallite dimensions (Table 1) and the rod-like shape correspond well to dimensions of crystallites from permanent human teeth documented by Daculsi and Kerebel $^{47}$ using TEM. HAP crystallites with similar morphology have been synthesized ${ }^{48}$ and were used for remineralization studies. ${ }^{21}$ However, other studies also report different morphologies such as spherical crystallites. ${ }^{36}$ While the morphology of individual crystallites in this study's HAP particles corresponds to that in enamel, their spatial arrangement is much more random in contrast to the highly ordered, directionally arranged crystallites in the prisms and interprismatic enamel (Figure 2(b)). Interestingly, neither the initial HAP powder nor the fraction adhering to the enamel samples after application as aqueous dispersion contained noteworthy amounts of free individual crystallites. The morphology of the crystallites (Figure 2(a)) did not change upon contact with water. Since HAP has the lowest solubility of all calcium phosphates, ${ }^{2}$ it is not surprising that it is inert to dissolution in this medium at neutral $\mathrm{pH}$ (Figures 7(b) and 7(d)). The particle clusters also remained intact, indicating that the bonding between the crystallites is stable enough to prevent their disintegration in water. Therefore, it is safe to assume that the prepared dispersions were free of nanoparticles, which is an important requirement for using them in oral care applications. The dipole properties of HAP and the resulting electrostatic forces have been postulated as being responsible for the cohesion of the crystallite clusters. ${ }^{49}$ However, the authors' high-resolution SEM images indicate that the majority of the crystallites are actually fused with their neighbors (Figure 2(a)), probably by the formation of mineral at their surfaces. This would also explain the absence of individual crystallites in the powder. If this would be the case, it must have happened already during the synthesis process. At present, the authors can only speculate about the nature of their bonding.

For the particle size distribution, the authors marked the particles on the SEM images manually, since the gray-scale contrast of the SEM images was not sufficient to obtain reliable particle recognition with automated image analysis tools such as ImageJ. While being more labor intensive, this procedure grants the best possible accuracy of the data. Subsequently, the authors calculated the planar projection area for each particle by using the analysis tool integrated in Photoshop. Since many particles are in contact with their neighbors, extreme care was taken to avoid the software recognizing multiple particles as one. However, this case cannot be fully excluded, and, therefore, a small systematic error has to be expected in the data. This affects mainly the analysis of the powder (Figure 3) due to the high density of particles in the images. The adhesion experiments are less affected. For the class of the largest particles with area projections between 14 and $1500 \mu \mathrm{m}^{2}$ in the powder, the authors sometimes observed that individual particles cluster to form larger aggregates (Figure 3(b)). In air, these particles adhere most probably by electrostatic forces. In some cases, it was difficult to discern whether a large particle consists of several smaller ones from the SEM images. The majority of the particles in the powder are in the size range between 100 and $200 \mathrm{~nm}$. Around this size class, the particle size forms a Gaussian distribution with the lower boundary at around $30 \mathrm{~nm}$ and the upper boundary at around $1 \cdot 3 \mu \mathrm{m}$ (Figure 3(c)). This size range corresponds well to the HAP microcluster size 
Quantitative affinity parameters of

synthetic hydroxyapatite and enamel

surfaces in vitro

Fabritius-Vilpoux, Enax, Herbig, Raabe and Fabritius reported from commercial mouth rinses in other studies (e.g. the study of Kensche et al. ${ }^{16}$ ).

Surface units are certainly not the ideal way to represent particle sizes, but must be considered the most accurate way given the limitation to two-dimensional data inherent to a study based on SEM analysis. The actual diameters that the authors present are a very rough approximation due to the irregular shape of the particles and should be considered as a help to get a more intuitive idea of the obtained data. However, surface units as a representation method is ideally suited to describe the correlation between HAP particle concentration and the degree of coverage achieved on this study's enamel test specimens. For application of HAP particle dispersions to the enamel surface samples, the authors developed a protocol that mimics the application of a mouth rinse. Controlled agitation of the dispersion through stirring and adjusting the position of the sample off-center and against the flow direction ensures that the type and direction of forces acting between particles and surface are comparable to those of the process happening in the mouth (Figure 1(d)). An exposure time of $1 \mathrm{~min}$ was also chosen according to the recommendations for commercial mouth rinse products. The data obtained under this study's experimental conditions clearly show a significant dose-response relationship: With increasing HAP particle concentration, the average coverage increases from $9.6 \%$ for $1 \%$ HAP to $13 \cdot 4 \%$ for $5 \%$ HAP and up to $32 \cdot 2 \%$ for $10 \%$ HAP of the total sample area (Figure 4). Against the authors' expectation, the increase in coverage is not linear and the difference between the 1 and 5\% dispersion concentrations is much smaller than the difference between the 5 and $10 \%$ dispersion concentrations. Since the enamel test specimens were pooled from different animals, teeth and tooth locations before being randomly distributed for the test series, differences in sample quality can be excluded as a reason for this. However, the authors observed great variations in the coverage density in individual samples (see Figures $\mathrm{S} 1-\mathrm{S} 9$ in the online supplementary material), which explains the large standard deviations of the results (Figure 4). With increasing HAP concentration, these variations become less pronounced and the coverage becomes more uniform (Figures S1-S9 in the online supplementary material). This is also reflected in the decrease in the standard deviation relative to the total area covered by particles from low to high concentration. It can thus be concluded that under this study's experimental conditions, the affinity of HAP particles toward enamel surfaces is not uniform. This study's results do not offer a concise explanation for this, since the surface quality of all used enamel test specimens was equally good. Possible reasons may be the differences in the orientation of the enamel crystallites at the surface, which result from the different angles at which the prisms were sectioned during polishing. This phenomenon manifests as different shapes of the prisms ranging from circular if cut perpendicularly to oval if cut obliquely (e.g. Figures 5(a)-5(c)). However, the authors did not observe a clear correlation between this and different particle density on the samples. Since a particular affinity of particles to attach to prism boundaries was not observed, these local depressions with inherently different crystallite arrangement can also be excluded. Despite this, the number of samples and particles analyzed for each concentration (15 areas on three different samples for the $1 \%\left(N_{\text {particles: }}: 2345\right)$ and 5\% HAP dispersions ( $\left.N_{\text {particles }}: 5912\right)$, nine areas on three different samples for the $10 \%$ HAP dispersion $\left(N_{\text {particles }}: 6006\right)$ ) is high enough to support confidently that an increase in particle concentration leads to an increase in coverage. At a HAP concentration of $10 \%$ (Figures 5(c) and S7-S9 in the online supplementary material), it is also observed that particles start to adhere to other particles instead of directly to the enamel as it was the case for the 1 and $5 \%$ concentrations (Figures 5(a) and 5(b) and S1-S6 in the online supplementary material).

From the viewpoint of possible remineralization effects, a cohesive monolayer of HAP particles in close contact with the enamel would be the most favorable condition. From the authors' observations, they hypothesize that, although not tested, concentrations of more than $10 \%$ HAP in mouth rinses would lead to the formation of particle clusters where those in the top layer cannot efficiently interact with the enamel anymore. Such particles would also easily be removed due to weak adhesion to other particles. Other effects of HAP have been shown to occur already at concentrations lower than $10 \%$. It has been reported that $5 \%$ HAP particles in a mouth rinse already efficiently reduce bacterial colonization on enamel samples in situ. ${ }^{16}$ Even far lower concentrations of HAP in the oral cavity have the potential to act as attachment sites for bacteria, thereby impeding their attachment to the tooth surface.

Analysis of the size distribution of the adhering particles (Figure 6) shows that virtually all of the size classes present in the powder (Figure 3(c)) do also adhere to the enamel in vitro with the exception of the very large ones with diameters above $4 \mu \mathrm{m}$. While slight differences are observed in the abundance of certain size classes for the different concentrations, the distributions follow the same trend. Solely the size classes with area projections larger than $0 \cdot 1 \mu \mathrm{m}^{2}$ are slightly less abundant after the experiment, but the differences compared to the powder are very small (1-2\%). However, this indicates that larger particles adhere less well than the smaller ones. The very large particles with area projections between 14 and $1500 \mu \mathrm{m}^{2}$ make up about $1 \%$ of the total number of particles in the powder that the authors use, but only around $0 \cdot 1 \%$ of the ones adhering to enamel. This indicates that there is a size limit for HAP particles above which they do not adhere to or remain attached to enamel surfaces. This limit is most probably defined by the adhesive forces between a particle and the enamel and the mass of the particle. Particles whose masses are larger than the adhesive forces at their contact area may adhere to the enamel while the sample is still in the aqueous dispersion, but will fall off when the sample is removed from the beaker or during the subsequent washing steps. Also, smaller particles have a larger contact area in relation to their mass than larger ones. This study's data suggest that for pure mineral-mineral interaction between 


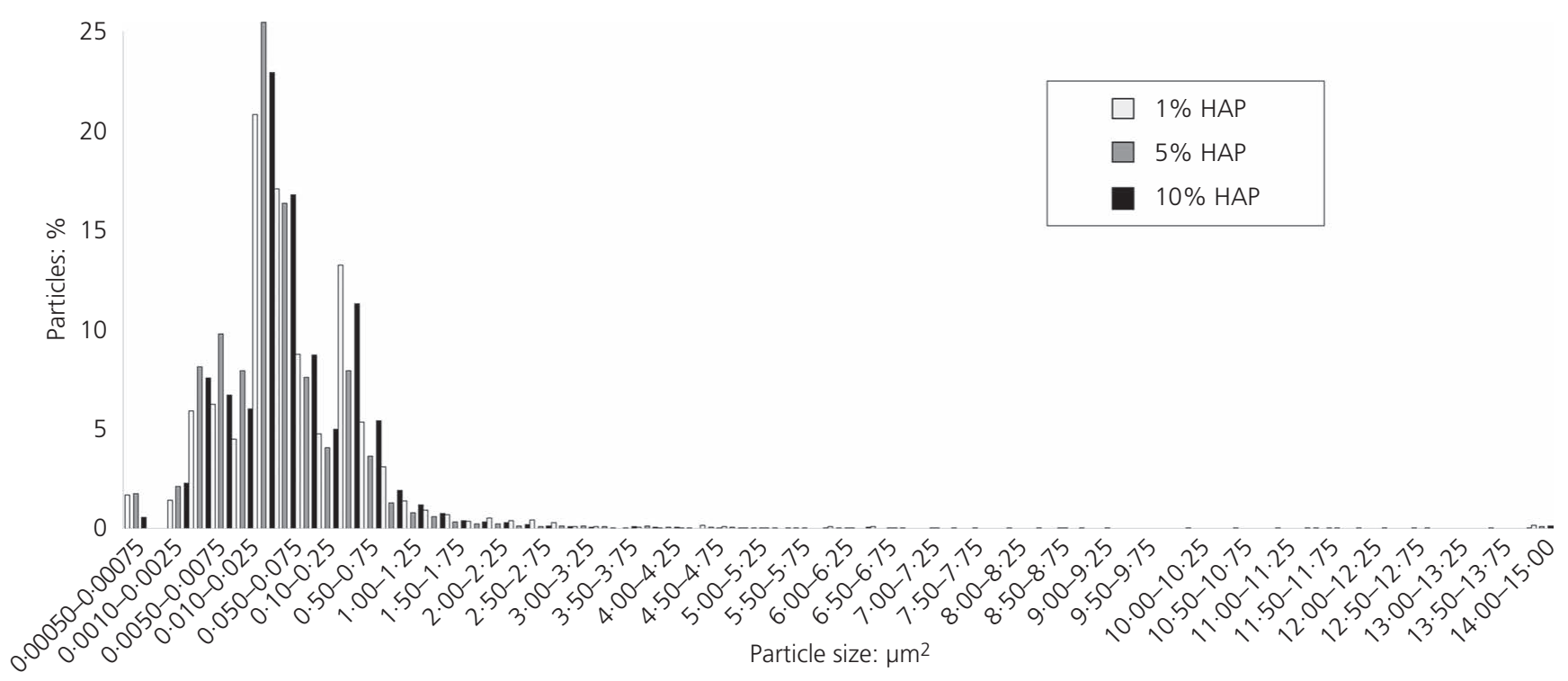

Figure 6. Quantitative analysis of the size distribution of HAP particles adhering to standardized bovine enamel surfaces after in vitro application. The very large particles are allotted to one class $\left(14-1500 \mu \mathrm{m}^{2}\right)$ for clarity of representation

HAP particles and bovine tooth enamel, this size boundary is at an area projection of about $1.25 \mu \mathrm{m}^{2}$ corresponding to about $1.3 \mu \mathrm{m}$ particle diameter assuming a spherical particle. In reality, this value may fluctuate since the irregularly shaped particles may have larger contact areas.

The question about the nature of the adhesive forces and their strength can unfortunately not be answered by this study. The presence of organic material that can act as adhesion promoter can be excluded as shown by high-resolution SEM images of particles on the enamel surface (Figures 7(a) and 7(b)). What can be said is that the particles certainly interact with the enamel surface through electrostatic forces, van der Waals forces and hydrogen bonds inherited from their chemical composition. Here, smaller particles will experience stronger bonding due to their higher surface charges ${ }^{49}$ and larger contact area with respect to their size. Another, although probably much smaller factor is mechanical interlocking due to the nanoscopic surface roughness caused by protruding, randomly oriented crystallites on the surface of HAP particles and the crystallites exposed by the etching step on the enamel surfaces (Figure 7). In future work, the authors plan to characterize experimentally the adhesion strength by measuring the forces necessary to separate particles from the surface using a nanoindenter. An interesting phenomenon that the authors observed in some of the samples was that HAP particles form connections with the enamel at their interface. These structures resemble outgrowths of individual crystallites that connect them and have similar widths (Figure 7(d)). Upon close inspection, these structures are equally stable in the electron beam as the HAP they connect. Therefore, it is very improbable that they are formed by organic residues that accumulated at the HAP particle-enamel interface during the sample drying process.
Another possibility is that these connections represent mineral bridges that have formed between crystallites from the particles and the enamel. In this case, they could have grown only through a local mineralization process where the ions originate from local dissolution processes and the HAP at the interface acts as nucleation site. This would suggest real chemical bonding through ionic bonds created by crystal growth and fusion, which would be a very interesting perspective of how remineralization could be explored for the optimization and development of new HAPbased oral care formulations. However, due to the low solubility of $\mathrm{HAP}^{2}$ it seems improbable that local dissolution processes occur to an extent that provides enough ions to enable the growth of such relatively large connections, and the water used for the dispersions was deionized. However, a recent study using atom probe tomography correlated with TEM has shown that human enamel crystallites are surrounded by about a $2 \mathrm{~nm}$ thick layer consisting of a magnesium-rich amorphous phosphate phase. ${ }^{50}$ Amorphous mineral phases are known to have a much higher solubility than crystalline ones. ${ }^{2,22}$ Should bovine enamel crystallites have a similar composition, this amorphous phase could very well be the ion donor for a mineralization process that fuses HAP particles and enamel crystallites. Since the superficial enamel crystallites in the authors' samples should be devoid of such an amorphous layer due to the phosphoric acid treatment step that was employed, the ions would have to come from a region below the surface. Due to the surface roughness of the treated enamel test specimens, this would be absolutely possible. However, the authors were only able to observe the connections where particles adhered to inclined enamel areas. This occurred only in samples where the dentin-enamel junction was exposed due to polishing of a concavely curved piece of tooth. To elucidate the nature of these connections definitely, the interface 
Bioinspired, Biomimetic and Nanobiomaterials Volume 8 Issue BBN2
Quantitative affinity parameters of synthetic hydroxyapatite and enamel surfaces in vitro

Fabritius-Vilpoux, Enax, Herbig, Raabe and Fabritius

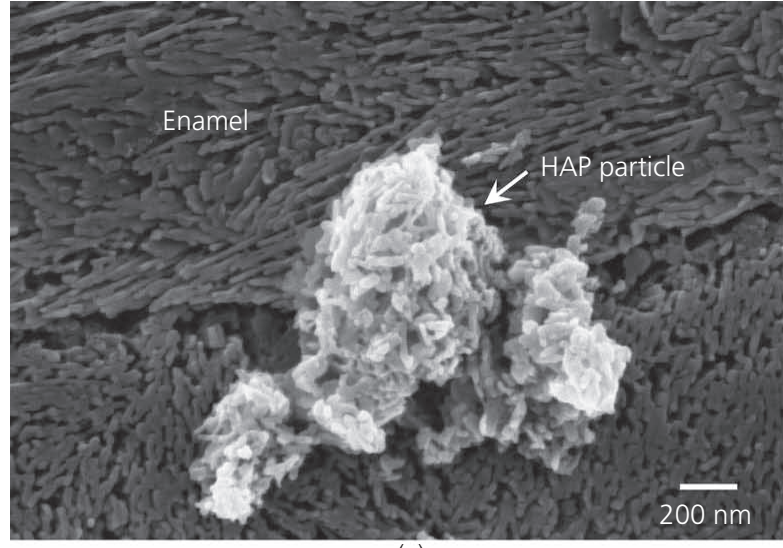

(a)

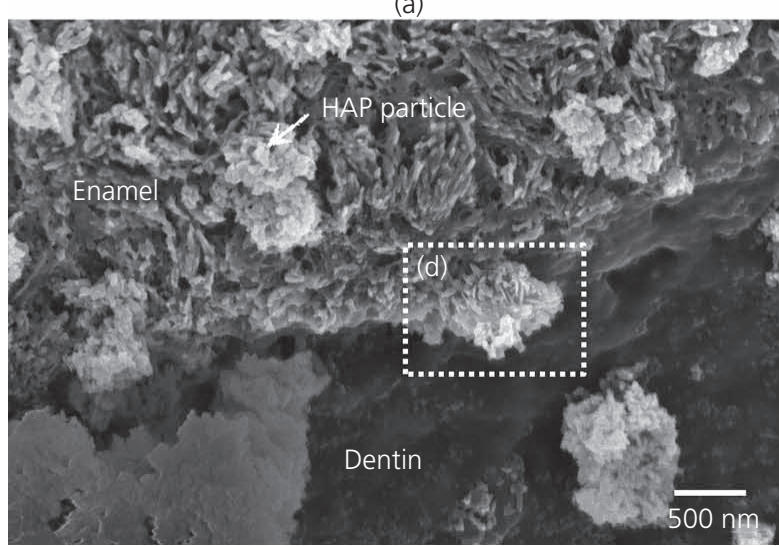

(c)

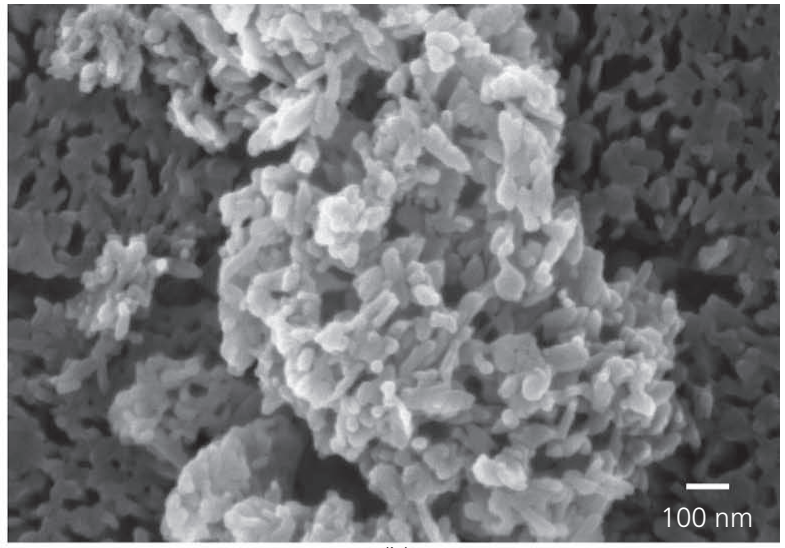

(b)

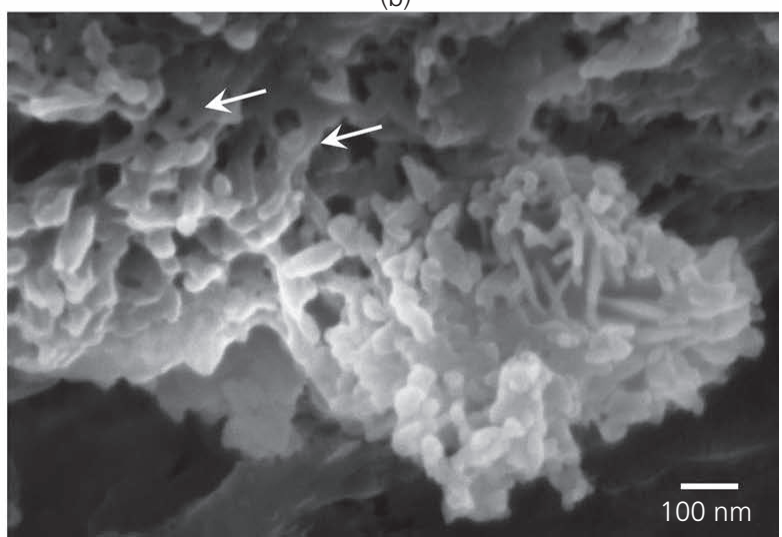

(d)

Figure 7. High-resolution electron micrographs showing the interfaces between polished and etched clean enamel surfaces and adhering HAP particles. (a) Particles are in immediate contact with the enamel surface. (b) Higher magnifications reveal the absence of substances that may act as adhesion promoter. (c) HAP particles adhering to the oblique enamel edge present at the dentin enamel junction. (d) Small solid bridges (arrows) connecting a HAP particle with the enamel crystallites at the interface

between particles and enamel has to be studied in cross-section using high-resolution TEM combined with elemental analysis, which will be subject of further work. The results can then be used to conduct numerical methods such as molecular dynamics simulations $^{51,52}$ that can help to improve the understanding of the adhesion process on the molecular scale in detail.

\section{Conclusions}

In this study, the authors present for the first time a comprehensive quantitative analysis of the interaction between pure synthetic HAP particles and clean enamel surfaces of bovine origin. It is shown that the HAP powder stock used qualifies as a biomimetic mineral due to the structural similarity between the crystallite clusters and enamel. The adhesion experiments demonstrate that there is a pronounced dose-response relationship with the analyzed enamel area coverage increasing from about 10 to over $30 \%$ with the concentration of the used HAP dispersion increasing from 1 to $10 \%$. In addition, it is shown that at $10 \%$ HAP concentration, the coverage is still largely a monolayer of particles. The particle size distribution shows that only particles with diameters up to about $1.3 \mu \mathrm{m}$ reliably adhere to the enamel surface, while the larger ones present in the powder do not. The authors discuss possible reasons for this and provide evidence for the presence of mineral bridges between crystallites at the interface between the HAP particles and the enamel surface. The data present a solid starting point for understanding the mineral-mineral interaction and are suitable as a base for optimization and refinement of HAP-based oral care formulations.

\section{Acknowledgements}

K.F.V. gratefully acknowledges financial support from Dr. Kurt Wolff GmbH \& Co. KG. The authors are thankful to Heidi Bögershausen from Max-Planck-Institut für Eisenforschung $\mathrm{GmbH}$ for valuable advice that helped optimize the enamel sample preparation.

\section{REFERENCES}

1. Lowenstam HA and Weiner S (1989) On Biomineralization. Oxford University Press, Oxford, UK.

2. Dorozhkin SV and Epple M (2002) Biological and medical significance of calcium phosphates. Angewandte Chemie International Edition 41: 3130-3146.

3. Berkovitz BKB, Boyde A, Frank RM et al. (1989) Teeth. SpringerVerlag, Berlin, Germany. 
Bioinspired, Biomimetic and Nanobiomaterials Volume 8 Issue BBN2
Quantitative affinity parameters of synthetic hydroxyapatite and enamel surfaces in vitro

Fabritius-Vilpoux, Enax, Herbig, Raabe and Fabritius
4. Moradian-Oldak J (2012) Protein-mediated enamel mineralization. Frontiers in Biosciences 17: 1996-2023.

5. Lussi A, Hellwig E and Klimek J (2012) Fluorides - mode of action and recommendations for use. Schweizer Monatsschrift für Zahnmedizin 122(11): 1030-1042.

6. ten Cate JM (2013) Contemporary perspective on the use of fluoride products in caries prevention. British Dental Journal 214: 161-167.

7. Craig RG, Welker D, Rothaut J et al. (2006) Dental materials. In Ullmann's Encyclopedia of Industrial Chemistry (Barbara E (ed.)). Wiley-VCH, Weinheim, Germany.

8. Wintermantel E and Ha SW (2008) Medizintechnik. Springer, Berlin, Germany.

9. Fejerskov O and Kidd E (2009) Dental Caries: the Disease and Its Clinical Management, 2nd edn. Blackwell Munksgaard, Copenhagen, Denmark.

10. Petersen PE, Bourgeois $D$, Ogawa $H$, Estupinan-Day $S$ and Ndiaye $C$ (2005) The global burden of oral diseases and risks to oral health. Bulletin of the World Health Organization 83: 661-669.

11. Lussi A and Ganss C (2014) Erosive Tooth Wear: from Diagnosis to Therapy, 2nd edn. Karger, Basel, Switzerland, vol. 20.

12. Enax J, Janus AM, Raabe D, Epple M and Fabritius HO (2014) Ultrastructural organization and micromechanical properties of shark tooth enameloid. Acta Biomaterialia 10: 3959-3968.

13. Luebke A, Enax J, Wey K et al. (2016) Composites of fluoroapatite and methylmethacrylate-based polymers (PMMA) for biomimetic tooth replacement. Bioinspiration and Biomimetics 11: 035001.

14. Brunton PA, Davies RP, Burke JL et al. (2013) Treatment of early caries lesions using biomimetic self-assembling peptides - a clinical safety trial. British Dental Journal 215: E6.

15. Hannig M and Hannig C (2010) Nanomaterials in preventive dentistry. Nature Nanotechnology 5: 565-569.

16. Kensche A, Holder C, Basche S et al. (2017) Efficacy of a mouthrinse based on hydroxyapatite to reduce initial bacterial colonisation in situ. Archives of Oral Biology 80: 18-26.

17. Niu LN, Zhang W, Pashley DH et al. (2014) Biomimetic remineralization of dentin. Dental Materials 30: 77-96.

18. Brown PW and Constantz B (1994) Hydroxyapatite and Related Materials. CRC Press, Boca Raton, FL, USA.

19. Amaechi BT and van Loveren C (2013) Fluorides and non-fluoride remineralization systems. Monographs of Oral Science 23: 15-26.

20. Enax J and Epple M (2018) Synthetic hydroxyapatite as a biomimetic oral care agent. Oral Health \& Preventive Dentistry 16: 7-19.

21. Roveri N, Battistella E, Foltran I et al. (2008) Synthetic biomimetic carbonate-hydroxyapatite nanocrystals for enamel remineralization. Advanced Materials Research 47: 821-824.

22. Meyer F, Amaechi BT, Fabritius HO and Enax J (2018) Overview of calcium phosphates used in biomimetic oral care. Open Dentistry Journal 12: 406-423.

23. Kani K, Kani M, Isozaki A et al. (1989) Effect of apatite-containing dentifrices on dental caries in school children. Journal of Dental Health 19: 104-109.

24. Meyer F and Enax J (2018) Early childhood caries: epidemiology, aetiology, and prevention. International Journal of Dentistry 1: 7-19.

25. Gillam DG (ed.) (2015) Dentine Hypersensitivity: Advances in Diagnosis, Management, and Treatment. Springer, Cham, Switzerland.

26. Orsini G, Procaccini M, Manzoli L et al. (2010) A double-blind randomized-controlled trial comparing the desensitizing efficacy of a new dentifrice containing carbonate/hydroxyapatite nanocrystals and a sodium fluoride/potassium nitrate dentifrice. Journal of Clinical Periodontology 37: 510-517.

27. Vano M, Derchi G, Barone A et al. (2018) Reducing dentine hypersensitivity with nano-hydroxyapatite toothpaste: a double-blind randomized controlled trial. Clinical Oral Investigations 22(1): $313-320$.
28. Hiller KA, Buchalla W, Grillmeier I, Neubauer C and Schmalz G (2018) In vitro effects of hydroxyapatite containing toothpastes on dentin permeability after multiple applications and ageing. Scientific Reports 8: 4888 .

29. Harks I, Jockel-Schneider Y, Schlagenhauf U et al. (2016) Impact of the daily use of a microcrystal hydroxyapatite dentifrice on de novo plaque formation and clinical/microbiological parameters of periodontal health: a randomized trial. PLOS One 11: e0160142.

30. Lelli M, Marchetti M, Foltran I et al. (2014) Remineralization and repair of enamel surface by biomimetic $\mathrm{Zn}$-carbonate hydroxyapatite containing toothpaste: a comparative in vivo study. Frontiers in Physiology 5: 333.

31. Kensche A, Pötschke S, Hannig C et al. (2016) Influence of calcium phosphate and apatite containing products on enamel erosion. Scientific World Journal 2016: 7959273.

32. Hannig C, Basche S, Burghardt T, Al-Ahmad A and Hannig M (2013) Influence of a mouthwash containing hydroxyapatite microclusters on bacterial adherence in situ. Clinical Oral Investigations 17: 805-814.

33. Dabanoglu A, Wood C, Garcia-Godoy F and Kunzelmann KH (2009) Whitening effect and morphological evaluation of hydroxyapatite materials. American Journal of Dentistry 22: 23-29.

34. Jin J, Xu X, Lai G and Kunzelmann KH (2013) Efficacy of tooth whitening with different calcium phosphate-based formulations. European Journal of Oral Sciences 121: 382-388.

35. Poggio C, Gulino C, Mirando M, Colombo M and Pietrocola G (2017) Protective effect of zinc-hydroxyapatite toothpastes on enamel erosion: an in vitro study. Journal of Clinical and Experimental Dentistry 9: e118-e122.

36. Shaffiey SR and Shaffiey SF (2016) Surface enamel remineralization by biomimetic nano hydroxyapatite crystals and fluoride ions effects. Journal of Ceramic Processing Research 17: 109-112.

37. Tschoppe P, Zandim DL, Martus P and Kielbassa AM (2011) Enamel and dentine remineralization by nano-hydroxyapatite toothpastes. Journal of Dentistry 39: 430-437.

38. Yamagishi K, Onuma K, Suzuki T et al. (2005) Materials chemistry: a synthetic enamel for rapid tooth repair. Nature 433: 819.

39. Huang SB, Gao SS and Yu HY (2009) Effect of nano-hydroxyapatite concentration on remineralization of initial enamel lesion in vitro. Biomedical Materials 4: 034104.

40. Najibfard K, Ramalingam K, Chedjieu I and Amaechi BT (2011) Remineralization of early caries by a nano-hydroxyapatite dentifrice. Journal of Clinical Dentistry 22: 139-143.

41. Esteves-Oliveira M, Meyer-Lueckel H, Wierichs RJ, Santos NM and Rodrigues JA (2017) Caries-preventive effect of anti-erosive and nano-hydroxyapatite-containing toothpastes in vitro. Clinical Oral Investigations 21: 291-300.

42. Walsh T, Worthington HV, Glenny AM et al. (2010) Fluoride toothpastes of different concentrations for preventing dental caries in children and adolescents. Cochrane Database of Systematic Reviews 2010(1): Cd007868.

43. LeGeros RZ (1981) Apatites in biological systems. Progress in Crystal Growth and Characterization of Materials 4: 1-45.

44. Teaford MF, Smith MM and Ferguson MWJ (eds) (2000) Development, Function and Evolution of Teeth. Cambridge University Press, Cambridge, UK.

45. Aydin B, Pamir T, Baltaci A, Orman MN and Turk T (2015) Effect of storage solutions on microhardness of crown enamel and dentin. European Journal of Dentistry 9: 262-266.

46. Esser M, Tinschert J and Marx R (1998) Materialkennwerte der Zahnhartsubstanz des Rindes im Vergleich zur humanen Zahnhartsubstanz. Deutsche Zahnärztliche Zeitschrift 53: 713-717 (in German).

47. Daculsi G and Kerebel B (1978) High-resolution electron microscope study of human enamel crystallites: size, shape, and growth. Journal of Ultrastructure Research 65: 163-172. 
Bioinspired, Biomimetic and Nanobiomaterials Volume 8 Issue BBN2
Quantitative affinity parameters of synthetic hydroxyapatite and enamel surfaces in vitro

Fabritius-Vilpoux, Enax, Herbig, Raabe and Fabritius
48. Enax J and Epple M (2018) Synthetic hydroxyapatite as a biomimetic oral care agent. Oral Health \& Preventive Dentistry 16: 7-19.

49. Bystrov V, Bystrova N, Dekhtyar Y et al. (2009) Size depended electrical properties of hydroxyapatite nanoparticles. In World Congress on Medical Physics and Biomedical Engineering, September 7-12, 2009, Munich, Germany (Dössel O and Schlegel WC (eds)). Springer, Berlin, Germany, vol. 25/8, pp. 230-232.

50. La Fontaine A, Zavgorodniy A, Liu H et al. (2016) Atomic-scale compositional mapping reveals $\mathrm{Mg}$-rich amorphous calcium phosphate in human dental enamel. Science Advances 2(9): e1601145.

51. Youssefian S, Liu P, Askarinejad S et al. (2015) Experimental and numerical measurements of adhesion energies between PHEMA and PGLYMA with hydroxyapatite crystal. Bioinspiration \& Biomimetics 10: 046011.

52. Shalchy F and Rahbar N (2015) Nanostructural characteristics and interfacial properties of polymer fibers in cement matrix. ACS Applied Materials \& Interfaces 7(31): 17278-17286.

\section{How can you contribute?}

To discuss this paper, please submit up to 500 words to the journal office at journals@ice.org.uk. Your contribution will be forwarded to the author(s) for a reply and, if considered appropriate by the editor-in-chief, it will be published as a discussion in a future issue of the journal.

ICE Science journals rely entirely on contributions from the field of materials science and engineering. Information about how to submit your paper online is available at www.icevirtuallibrary.com/page/authors, where you will also find detailed author guidelines. 